\title{
A TRANSCENDENTAL UNBOUNDED CONTINUED FRACTION EXPANSIONS OVER A FINITE FIELD
}

\author{
R. Ghorbel ${ }^{1}$, H. Kthiri ${ }^{2}$ \\ Faculty of science of Sfax, BP 1171, 3000 Sfax, Tunisia, emails: \\ ${ }^{1}$ rimaghorbel@yahoo.fr, ${ }^{2}$ hassenkthiri@gmail.com
}

\begin{abstract}
Let $\mathbb{F}_{q}$ be a finite field and $\mathbb{F}_{q}\left(\left(X^{-1}\right)\right)$ the field of formal power series with coefficients in $\mathbb{F}_{q}$. The purpose of this paper is to exhibit a family of transcendental continued fractions of formal power series over a finite field through some specific irregularities of its partial quotients.
\end{abstract}

Key words and Phrases: Continued fraction, formal power series, transcendence, finite fields.

\section{INTRODUCTION}

In $[5,1]$, Maillet and Baker studied the real number $x=\left[a_{0}, a_{1}, \ldots\right]$ where $\left(a_{i}\right)_{i \geq 0}$ is the sequence of partial quotients of $x$ such that $a_{n}=a_{n+1}=\ldots=$ $a_{n+\lambda(n)-1}$, for infinitely many positive integers $n$ where $\lambda(n)$ is a sequence of integers verifying some increasing properties. The authors showed that such an $x$ is transcendental and their proof is based on the theorem of Davenport and Roth [7] and the consideration of the assumption on $\left(a_{i}\right)_{i \geq 0}$ that it is bounded.

Later, Adamczewski and Bugeaud [3] suggested a new transcendence criteria for continued fractions by using the Schmidt subspace Theorem given in [15] where the author showed that if an irrational number is very well approximated by quadratic numbers then it is quadratic or transcendental.

Unfortunately, in the field of formal series, we do not have similar theorems to those of Roth and Schmidt. In 1976, Baum and Sweet [8] proved that the unique solution in $\mathbb{F}_{2}\left(\left(X^{-1}\right)\right)$ of the cubic equation

$$
X \alpha^{3}+\alpha+X=0
$$

has a continued fraction expansion with partial quotients of bounded degree. They observed that no real algebraic number of degree $\geq 3$ has yet been shown to

2020 Mathematics Subject Classification: 11R06.

Received: 11-05-2019, accepted: 26-02-2021. 
have bounded or unbounded partial quotients.

In 1986, Mills and Robbins [14] provided an example of algebraic formal series over $\mathbb{F}_{2}(X)$ whose sequence of partial quotients is unbounded.

In 2004, Mkaouar [12] gave a similar result to the Baker one [1] concerning the transcendence of formal series over a finite field.

In 2006, Hbaib, Mkaouar and Tounsi [10] proved a result which allows the construction of a family of transcendental continued fractions over $\mathbb{F}_{q}\left(\left(X^{-1}\right)\right)$ from an algebraic formal series of degree more than 2 .

In 2019, in collaboration with S. Driss [9], we showed that, if the continued fraction of a formal power series in $\mathbb{F}_{q}\left(\left(X^{-1}\right)\right)$ begins with sufficiently large geometric blocks, then $\mathrm{f}$ is transcendental.

In this work we give a new transcendence criteria which depends only on the length of specific blocks appearing in the sequence of partial quotients.

This article is organized as follows: In the next section, we set up the problem and we give some useful definitions and known results in the field of formal power series and the continued fraction expansions over this field. In section 3 , we treat the objective of this paper and establish a new general result on a transcendence criterion. Later, we give an example to illustrate the importance of our result.

\section{FIELD OF FORMAL SERIES $\mathbb{F}_{q}\left(\left(X^{-1}\right)\right)$}

Let $\mathbb{F}_{q}$ be a field with $q>1$ elements of characteristic $p>0$. We denote by $\mathbb{F}_{q}[X]$ the ring of polynomials with coefficients in $\mathbb{F}_{q}$ and $\mathbb{F}_{q}(X)$ the field of rational functions. Let $\mathbb{F}_{q}\left(\left(X^{-1}\right)\right)$ be the field of formal series, i.e., for any $f \in \mathbb{F}_{q}\left(\left(X^{-1}\right)\right)$, $f$ can be written as,

$$
f=\sum_{n \geq n_{0}} b_{n} X^{-n}
$$

where $b_{n} \in \mathbb{F}_{q}$ and $n_{0} \in \mathbb{Z}$. A formal series $f=\sum_{n \geq n_{0}} b_{n} X^{-n}$ has a unique decomposition as $f=[f]+\{f\}$ with the polynomial part $[f] \in \mathbb{F}_{q}[X]$ and the fractional part verifying $|\{f\}|<1$. Here we define the non-archimedean absolute value as follows:

$$
|f|= \begin{cases}q^{\operatorname{deg} f} & \text { if } f \neq 0 \\ 0 & \text { if } f=0\end{cases}
$$


Thus, $|f+g| \leq \max (|f|,|g|)$ and $|f+g|=\max (|f|,|g|)$ if $|f| \neq|g|$. Let us also recall that the continued fraction expansion of a formal series $f$ is written as

$$
f=a_{0}+\frac{1}{a_{1}+\frac{1}{a_{2}+\frac{1}{\ddots}}}=\left[a_{0}, a_{1}, a_{2}, \ldots\right],
$$

where $a_{0}=[f]$, and for $i \geq 1, a_{i}=\left[f_{i}\right] \in \mathbb{F}_{q}[X]$ with $\operatorname{deg}\left(a_{i}\right) \geq 1$ and $f_{i}=\frac{1}{\left\{f_{i-1}\right\}}$. Here, $\left(a_{i}\right)_{i \geq 0}$ is called the sequence of partial quotients of $\mathrm{f}$ and we denote by $f_{n}=\left[a_{n}, a_{n+1}, \ldots\right]$ the $\mathrm{n}$-th complete quotient of $f$.

Remark 2.1. [4]

(1) If $\left(\operatorname{deg}\left(a_{i}\right)\right)_{i \geq 0}$ is bounded then $f$ has a bounded continued fraction expansion.

(2) The expansion is finite if and only if $f \in \mathbb{F}_{q}(X)$.

(3) The sequence of partial quotients of $f$ is ultimately periodic if and only if $f$ is quadratic over $\mathbb{F}_{q}(X)$.

Now, we define two sequences of polynomials $\left(P_{n}\right)_{n \geq 0}$ and $\left(Q_{n}\right)_{n \geq 0}$ by

$$
\begin{gathered}
P_{0}=a_{0}, Q_{0}=1, P_{1}=a_{0} a_{1}+1, Q_{1}=a_{1} \\
P_{n}=a_{n} P_{n-1}+P_{n-2}, Q_{n}=a_{n} Q_{n-1}+Q_{n-2}, \text { for } n \geq 2 .
\end{gathered}
$$

We can check that

$$
\begin{gathered}
P_{n} Q_{n-1}-P_{n-1} Q_{n}=(-1)^{n-1}, \text { for } n \geq 1, \\
\frac{P_{n}}{Q_{n}}=\left[a_{0}, a_{1}, \ldots, a_{n}\right], \text { for } n \geq 0 .
\end{gathered}
$$

$\frac{P_{n}}{Q_{n}}$ is called the $n^{t h}$ convergent of $f$ and it satisfies

$$
\lim _{n \rightarrow \infty} \frac{P_{n}}{Q_{n}}=f=\left[a_{0}, a_{1}, \ldots, a_{n}, \ldots\right] .
$$

According to the properties of on-archimedean absolute value, we find the following important equality

$$
\left|f-\frac{P_{n}}{Q_{n}}\right|=\left|\frac{P_{n+1}}{Q_{n+1}}-\frac{P_{n}}{Q_{n}}\right|=\left|Q_{n} Q_{n+1}\right|^{-1}=\left|a_{n+1}\right|^{-1}\left|Q_{n}\right|^{-2} .
$$

Let $f$ be an algebraic formal series of minimal polynomial $P(Y)=A_{m} Y^{m}+$ $A_{m-1} Y^{m-1}+\ldots+A_{0}$ where $A_{i} \in \mathbb{F}_{q}[X]$. We let $H(f)=\max _{0 \leq i \leq m}\left|A_{i}\right|$ and $\sigma(f)=A_{m}$. 


\section{Results}

Before giving the main result, we need to introduce some notation.

If $K=u_{\alpha_{0}} u_{\alpha_{1}} \ldots u_{\alpha_{n}}$ is a finite block formed by $n+1$ polynomials, we denote by $|K|$ the length of this block and by $\varphi(K)$ the maximal degree which appears in the terms of $K$, which means that $\varphi(K)=\max _{0 \leq i \leq n}\left(\operatorname{deg}\left(u_{\alpha_{i}}\right)\right)$.

If $U_{n}, V_{n}$ are two finite blocks of polynomials, we write $U_{n} V_{n}$ for the block resulting by concatenation of these two blocks.

Definition 3.1. We say that $U$ is geometric of order $s$ if there exists $K_{i}$ such that $U=K_{i} K_{i}^{q} K_{i}^{q^{2}} \cdots K_{i}^{q^{s-1}}$ with $K_{i}=H_{1}^{(i)} \ldots H_{n}^{(i)}$ for each $i \geq 0$ being a block of polynomials.

Now, we present the most important result, interesting in its own right, of our paper which severs to provide a criterion for a given formal power series being transcendental.

Theorem 3.2. Let $f \in \mathbb{F}_{q}\left(\left(X^{-1}\right)\right)$ such that $f=\left[U_{0} V_{0}, U_{1} V_{1}, \ldots, U_{n} V_{n}, \ldots\right]$ where $\left(U_{n}\right)_{n \geq 0}$ and $\left(V_{n}\right)_{n \geq 0}$ are two sequences of finite blocks of polynomials such that

(1) $U_{i}$ is geometric of order $\lambda_{i}$.

(2) $\left(\lambda_{i}\right)_{i \geq 1}$ is an increasing sequence of positive integers.

(3) The sequences $\left(\frac{\left|V_{n}\right|}{\left|U_{n}\right|}\right)_{n \geq 0}$ and $\left(\frac{\left|U_{n}\right|}{\lambda_{n}}\right)_{n \geq 0}$ are bounded.

(4) $\sup \left|H_{i}^{(j)}\right|<M \quad \forall i \quad \forall j$

(5) $\varphi\left(V_{n}\right) \leq \varphi\left(U_{n}\right)$, for all $n \geq 0$.

If $f$ satisfies

$$
\limsup _{n \rightarrow+\infty} \frac{q^{\lambda_{n}}-1}{n \lambda_{n-1} q^{\lambda_{n-1}}}=+\infty,
$$

then $f$ is transcendental.

To prove Theorem 3.2 we will need the following lemmas.

Lemma 3.3. [10] Assume that $f$ is an algebraic formal series of degree $d$ such that $f=\left[a_{1}, a_{2}, \ldots, a_{t}, h\right]$ where for any $1 \leq i \leq t, a_{i} \in \mathbb{F}_{q}[X], h \in \mathbb{F}_{q}\left(\left(X^{-1}\right)\right)$. If $|f| \geq 1$ and $|h|>1$ then $h$ is algebraic of degree $d$ and

$$
H(h) \leq H(f)\left|\prod_{i=1}^{t} a_{i}\right|^{d-2} .
$$

Lemma 3.4. [?, Lemma 2.2] Let $P(Y)=A_{m} Y^{m}+A_{m-1} Y^{m-1}+\ldots+A_{0}$ be a reduced polynomial in $\mathbb{F}_{q}[X][Y]$ with $A_{i} \in \mathbb{F}_{q}[X], A_{m} \neq 0$ and $\left|A_{m-1}\right|>\left|A_{i}\right|$ for all $i \neq m-1$. Then $P$ admits a unique root $f$ with $|f|>1$ and $[f]=\left[-\frac{A_{m-1}}{A_{m}}\right]$. Moreover, $P$ is irreducible. 
Lemma 3.5. [13] Let $f=\left[a_{0}, a_{1}, \ldots\right]$ and $g=\left[b_{0}, b_{1}, \ldots\right]$ be two formal series having the same first $n+1$ terms of partial quotients. Then

$$
|f-g| \leq \frac{1}{\left|Q_{n}\right|^{2}}
$$

where $\left(Q_{n}\right)_{n \geq 0}$ is a sequence of polynomials which is defined in (2).

Lemma 3.6. [10] Let $f$ and $g$ be two algebraic formal series of degree $d$ and $m$ respectively. If $g$ is reduced and $f \neq g$, then

$$
|f-g| \geq \frac{1}{H(f)^{m}|g|^{d-2}|\sigma(g)|^{\max (m-1, m(d-m+2)-1)}} .
$$

Proof. of Theorem 3.2 Assume that $f$ is algebraic of degree $d>2$ and $U_{i}=$ $K_{i} K_{i}^{q} K_{i}^{q^{2}} \cdots K_{i}^{q^{\lambda_{i}-1}}$, for any $i \geq 0$, with $K_{i}=H_{1}^{(i)} \cdots H_{\beta_{i}}^{(i)} \in F_{q}[X]$ of degree $\geq 1$.

Let us use the notation: $K_{n}=H_{1}^{(n)} \ldots H_{\beta_{n}}^{(n)} \in \mathbb{F}_{q}[X],\left|K_{n}\right|=\beta_{n},\left|V_{n}\right|=s_{n}$, $\left|U_{n}\right|=\lambda_{n} \beta_{n}$ for all $n \geq 0$ and $\alpha_{n}=\sum_{i=0}^{n-1}\left(\lambda_{i} \beta_{i}+s_{i}\right)$.

Let $g_{n}$ denote the continued fraction $\left[U_{n}, U_{n}^{q}, U_{n}^{q^{2}}, \ldots, U_{n}^{q^{s}}, \ldots\right]$. An easy calculation ensures that $g_{n}$ verifies the following equation

$$
q_{\beta_{n}} g_{n}^{q+1}-p_{\beta_{n}} g_{n}^{q}+q_{\beta_{n}-1} g_{n}-p_{\beta_{n}-1}=0,
$$

where $\left(\frac{p_{n}}{q_{n}}\right)_{n \geq 1}$ is the sequence of reduit of $g_{n}$. Hence Lemma 3.4 guarantees that $g_{n}$ is algebraic of degree $q+1$ such that $H\left(g_{n}\right)=\left|p_{\beta_{n}}\right|=\prod_{i=1}^{\beta_{n}}\left|H_{i}^{(n)}\right|$ and $\left|\sigma\left(g_{n}\right)\right|=\left|q_{\beta_{n}}\right|=\prod_{i=2}^{\beta_{n}}\left|H_{i}^{(n)}\right|$.

Let $f_{\alpha_{n}}=\left[U_{n} V_{n}, U_{n+1} V_{n+1}, \ldots\right]$ denote the $\alpha_{n}^{\text {th }}$ complete quotient of $f$. Since $\sup \left|H_{i}^{(j)}\right|<M, \quad \forall i \quad \forall j$, then for sufficiently large $n, g_{n} \neq f_{\alpha_{n}}$. On the other hand, it follows from Lemma 3.3 that $f_{\alpha_{n}}$ is algebraic of degree $d>2$. Therefore, according to Lemma 3.6, we can check, for sufficiently large $n$ that:

$$
\left|f_{\alpha_{n}}-g_{n}\right| \geq \frac{1}{H\left(f_{\alpha_{n}}\right)^{q+1}\left|g_{n}\right|^{d-2}\left|\sigma\left(g_{n}\right)\right|^{d(q+1)-q^{2}}} .
$$

So

$$
\left|f_{\alpha_{n}}-g_{n}\right| \geq \frac{1}{H\left(f_{\alpha_{n}}\right)^{q+1}\left|g_{n}\right|^{d-2}\left|\prod_{i=2}^{\beta_{n}} H_{i}^{(n)}\right|^{d(q+1)-q^{2}}} .
$$

Moreover, by using Lemma 3.3, we can check that $H\left(f_{\alpha_{n}}\right) \leq H(f)\left|\prod_{i=1}^{\alpha_{n}} a_{i}\right|^{d-2}$, where $\left(a_{i}\right)_{i \geq 0}$ is the sequence of partial quotients of $f$.

So

$$
\left|f_{\alpha_{n}}-g_{n}\right| \geq \frac{1}{H(f)^{q+1}\left|\prod_{i=1}^{\alpha_{n}} a_{i}\right|^{(d-2)(q+1)}\left|H_{1}^{(n)}\right|^{d-2}\left|\prod_{i=2}^{\beta_{n}} H_{i}^{(n)}\right|^{d(q+1)-q^{2}}} .
$$


Furthermore, $f_{\alpha_{n}}$ and $g_{n}$ have the same first $\lambda_{n}$ partial quotients, hence Lemma 3.5 implies that

$$
\left|f_{\alpha_{n}}-g_{n}\right| \leq \frac{1}{\left|H_{1}^{(n)} \ldots H_{\beta_{n}}^{(n)}\right|^{2\left(\frac{q^{\lambda} n-1}{q-1}\right)}} .
$$

Combining (6) and (7), we get

$$
H(f)^{q+1}\left|\prod_{i=1}^{\alpha_{n}} a_{i}\right|^{(d-2)(q+1)}\left|H_{1}^{(n)}\right|^{d-2}\left|\prod_{i=2}^{\beta_{n}} H_{i}^{(n)}\right|^{d(q+1)-q^{2}} \geq\left|H_{1}^{(n)} \ldots H_{\beta_{n}}^{(n)}\right|^{2\left(\frac{q^{\lambda} n-1}{q-1}\right)} .
$$

Let $M=\sup _{i \geq 0}\left|\left(H_{j}^{(i)}\right)\right|$ for all $1 \leq j \leq n$. Using the fact that $\varphi\left(V_{i}\right) \leq \varphi\left(U_{i}\right)$ for all $i \geq 0$, we get $\operatorname{deg}\left(a_{i}\right) \leq M^{q^{\lambda_{n-1}-1}}$, for all $0 \leq i \leq \alpha_{n}$. Then

$$
\left|\prod_{i=1}^{\alpha_{n}} a_{i}\right|=\prod_{i=1}^{\alpha_{n}} q^{\operatorname{deg} a_{i}} \leq \prod_{i=1}^{\alpha_{n}} M^{q^{\lambda_{n}-1}-1}=M^{\left(q^{\lambda_{n}-1-1}\right) \alpha_{n}}
$$

So

$$
\left.H(f)^{q+1} M^{\left(q^{\lambda} n-1-1\right.}\right) \alpha_{n}(d-2)(q+1) M^{(d-2)} M^{\left(\beta_{n}-1\right)\left(d(q+1)-q^{2}\right)} \geq q^{\frac{2 \beta_{n}\left(q^{\lambda} n-1\right)}{q-1}} .
$$

From this we get

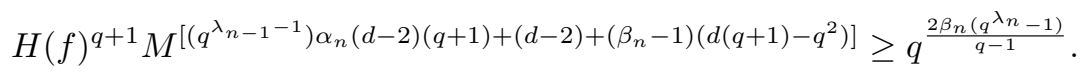

So

$$
\begin{gathered}
(q+1) \log (H(f))+\left[\left(q^{\lambda_{n-1}-1}\right) \alpha_{n}(d-2)(q+1)+(d-2)+\left(\beta_{n}-1\right)\left(d(q+1)-q^{2}\right)\right] \log (M) \\
\geq \frac{2 \beta_{n}\left(q^{\lambda_{n}}-1\right)}{q-1} \log (q) .
\end{gathered}
$$

Using the fact that $\left|K_{n}\right|=\beta_{n}$ is bounded and tend $n$ to $\infty$,

$$
\left(q^{\lambda_{n-1}-1}\right) \alpha_{n}(d-2)(q+1) \log (M) \geq \frac{2 \beta_{n}\left(q^{\lambda_{n}}-1\right)}{q-1} \log (q) .
$$

Therefore

$$
\limsup _{n \rightarrow+\infty} \frac{q^{\lambda_{n}}-1}{\alpha_{n} q^{\left(\lambda_{n-1}-1\right)}} \leq \frac{(d-2)\left(q^{2}-1\right) \log (M)}{2 \log (q) \beta_{n}} \leq C,
$$

with $C=\frac{(d-2)\left(q^{2}-1\right) \log (M)}{2 \log (q)}$.

Since the sequence $\left(\frac{\left|V_{i}\right|}{\left|U_{i}\right|}\right)_{i \geq 0}$ is bounded, there exists $c>0$ such that $s_{i}<c \lambda_{i}$ for all $i \geq 0$. Also, since $\left(\frac{\left|U_{n}\right|}{\lambda_{n}}\right)_{n \geq 0}$ is bounded, then there is a real $k>0$ such that 
$\frac{\left|U_{n}\right|}{\lambda_{n}}=\beta_{n} \leq k$, for all $n \geq 0$.

Thus

$$
\alpha_{n}<(c+1) \lambda_{n-1} n \beta_{n} \leq k(c+1) \lambda_{n-1} n=h \lambda_{n-1} n
$$

with $h=(c+1) k$. Hence, we conclude that

$$
\limsup _{n \rightarrow+\infty} \frac{q^{\lambda_{n}}-1}{n \lambda_{n-1} q^{\lambda_{n-1}}}<\infty
$$

which contradicts our hypothesis.

We close this paper with the following application.

Example 3.7. Let $f \in \mathbb{F}_{q}\left(\left(X^{-1}\right)\right)$ for $q=p^{a}$ ( $p$ a prime, $a \geq 1$ an integer) such that $f=\left[U_{0} V_{0}, U_{1} V_{1}, \cdots, U_{n} V_{n}, \cdots\right]$

where $U_{i}=\left[H_{i}, H_{i}^{2}, H_{i}^{4}, \cdots, H_{i}^{2^{\lambda_{i}-1}}\right]$, with $H_{i}=\left[P_{i}^{(1)}, P_{i}^{(2)}, \cdots, P_{i}^{(s)}\right]$ such that $P_{i}^{(j)}=X^{j}+i$ and $V_{i}=\left[X, X^{2}, X, X^{2}, \cdots, X, X^{2}\right]$ of length $\lambda_{i}=(i+1)^{2}$, for all $i \geq 0$. Then $f$ is transcendental because

$$
\limsup _{n \rightarrow+\infty} \frac{q^{(n+1)^{2}}-1}{n^{3} \cdot q^{n^{2}}}=+\infty .
$$

\section{REFERENCES}

[1] A. Baker. , "Continued fractions of trascendental numbers", Mathematika., 9 (1962), 1-8.

[2] A. Khintchine., "Continued fractions", Gosudarstv. Izdat. Tech-Teor. Lit. MoscowLeningrad., $2^{\text {nd }}$ edition, 1949, (In Russian).

[3] Adamczewski and Y. Bugea., "On the Maillet-Baker continued fractions", J. Reine Angew. Math., 606 (2007), 105-121.

[4] B. Ammous, S. Driss and M. Hbaib., "Continued Fractions and Transcendence of Formal Power Series Over a Finite Field,", Mediterranean Journal of Mathematics, 13 (2016), 527536.

[5] E. Maillet., "Introduction la théorie des nombres transcendants et des proprits arithmtiques des fonctions", Gauthier-Villars., Paris, 1906.

[6] J. Liouville., "Sur des classes trs tendues de quantits dont la valeur n'est ni algbrique ni même rductibles des rationnelles algbriques", J. Math. Pures Appl., 16 (1851), 133-142.

[7] H. Davenport and K. F. Roth., "Rational approximations to algebraic numbers", Mathematika. 2 (1955), 160-167.

[8] L.E. Baum and H.M. Sweet., "Continued fractions of algebraic power series in characteristic 2", Ann. Math., 103 (1976), 593-610.

[9] S. Driss and H. Kthiri., "On the geometric continued fraction in positive charateristic", J. Indones. Math. Soc., 25 (2019), 139-145.

[10] M. Hbaib, M. Mkaouar and K. Tounsi, " Un critre de transcendance dans le corps des séries formelles $\mathbb{F}_{q}\left(\left(X^{-1}\right)\right)$ ", J. Number Theory, 116 (2006), 140-149.

[11] M. Mkaouar., "Fractions continues et séries formelles algébriques réduites", Port. Math., 58 (2001).

[12] M. Mkaouar., "Transcendance de certaines fractions continues dans le corps des séries formelles", J. Algebra., 281 (2004), 502-507. 
[13] O. Perron., "Die Lehre von den Kettenbrüchen", Teubner, Leipzig, 1929.

[14] W.H. Mills and D.P. Robbins, "Continued fractions for certain algebraic power series", J. Number Theory, 23 (1986), 388-404.

[15] W.M. Schmidt., "Diophantine approximation", Lecture Notes in Mathematics, vol. 785. Springer, Berlin (1980). 